\title{
DESIGN AND IMPLEMENTATION OF THE MEDIUM-BETA INSERT OF THE FERMILAB RECYCLER RING*
}

\author{
W. Wan, ORNL, Oak Ridge, TN 37831, USA \\ C. Gattuso, D. E. Johnson, C. S. Mishra, J. A. Volk, FNAL, Batavia, IL 60510, USA
}

\begin{abstract}
The design of the newly installed medium- $\beta$ insert of the Fermilab Recycler Ring is presented. The design philosophy is outlined. The stringent optical and physical constraints, as well as their influence on the design, are discussed. The impact of the medium- $\beta$ insert on the Recycler Ring is shown. Engineering design and installation of the new insert is presented.
\end{abstract}

\section{INTRODUCTION}

The Recycler Ring being commissioned at Fermilab [1] is a permanent magnet storage ring designed to recycle used anti-protons from the Tevatron and hence further increase the luminosity [2] [3]. In order to reuse the antiprotons after a store, they have to be cooled in the Recycler Ring, which is to be achieved mainly through electron cooling [4]. To accommodate electron cooling, a high- $\beta$ insert (Figure 1) was designed and installed in the RR30 long straight section [5] [1]. Recently, the goal of electron cooling was shifted from transverse cooling at the expense of longitudinal cooling to roughly equal cooling in all dimensions. As a result, the desired $\beta$ functions in the cooling channel were reduced from 200 $\mathrm{m}$ to $24 \mathrm{~m}$ and the length of the channel from $\sim 80 \mathrm{~m}$ to $\sim 20 \mathrm{~m} \mathrm{[6]}$.

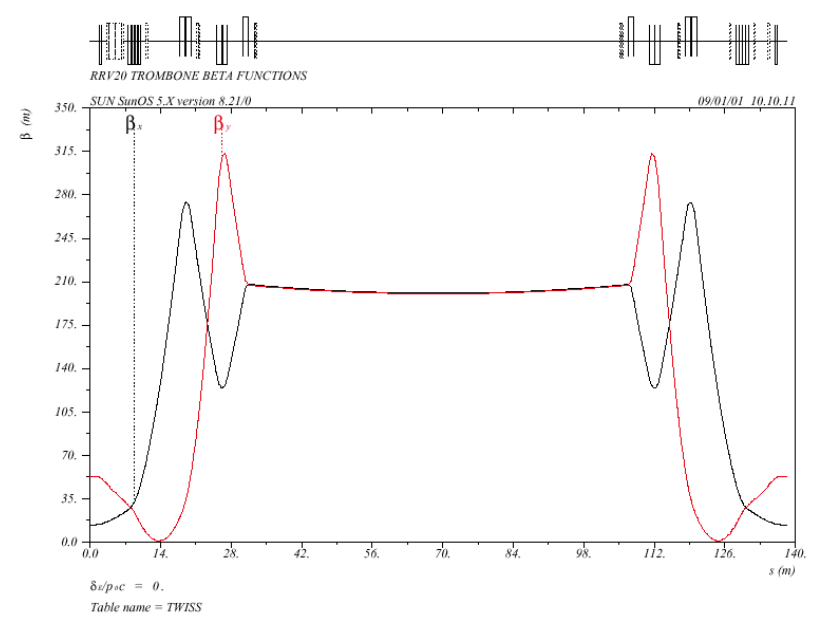

Figure 1: $\beta$-function plot of the high- $\beta$ insert

An attempt on a new design was made by A. Burov [6], who presented a lattice using most of the magnets in the

\footnotetext{
*Work supported by the U.S. Department of Energy under contract No. DE-AC02-76CHO3000.
}

RR30 straight. Yet it is estimated that it will take 2 to 3 months of down time to install the proposed insert. The whole process includes removing, cooling down, modifying and installing the magnets. In this paper, an alternative design is presented. It uses only 8 magnets in the RR30 straight, which leads to the reduction of down time to 3 week. Other quads in this design are the 32 quads designated for the permanent magnet phase trombone, which were stored in the Fermilab magnet factory. It is shown below that the new lattice meets all optical and physical constraints (Section 2) and has been successfully installed (Section 3).

\section{LATTICE}

The lattice was a compromise between the demand on optics and the constraints from the physical world. From the optical point of view, the insert has to be matched to the rest of the ring $\left(\beta_{\mathrm{x}}=12.45 \mathrm{~m}, \beta_{\mathrm{y}}=53 \mathrm{~m}, \alpha \mathrm{x}=\alpha \mathrm{y}=\right.$ 0 ), maintains the original fractional tunes $(0.425,0.415)$, keeps the maximum $\beta$ under control $(\beta \max <100 \mathrm{~m})$ and produces medium $\beta$-functions at the midpoint of the electron cool straight $\left(\beta_{X}=\beta_{y}=24 \mathrm{~m}, \alpha_{X}=\alpha_{y}=0\right)$. The following physical constraints played a role in the designing process. The first one is that the total length of the insert is fixed $(\sim 140 \mathrm{~m})$. The second one is that the length of the electron cooling is fixed ( $28 \mathrm{~m}$ drift space between magnets). The third constraint has a significant impact on the layout of the insert. It is that the two ends of the electron cooling straight have to be outside the MI30 so that the penetration can avoid drilling through concrete foundation of the building. Building MI30, which is about 30 meters long, sits in the middle of the insert. Since the electron cooling straight of the high- $\beta$ insert is longer than the build, it can be placed in the middle and both ends are on different sides of the building. As a result, the lattice of the high- $\beta$ insert is symmetric. In case of the medium- $\beta$ insert, the cooling straight is shorter than the building. Therefore the entire straight has to be on either side of the building, which breaks the symmetry of the lattice. The fourth constraint is that it is preferred to have as few Main Injector quads under the cooling straight as possible, so that the effect of the stray magnetic field from the Main Injector can be minimized. Since the spacing of the Main Injector quads $(17.29 \mathrm{~m})$ is smaller than the length of the cooling straight but greater than half its length, there is at least 1 Main Injector quad under the cooling straight. The last constraint is that gradient of the magnets should range 
between $75 \%$ and the full strength of the existing magnets.

The general design philosophy of the lattice is to use symmetry as much as possible to minimize the number of quad families. Since overall symmetry is ruled out, the lattice is divided into two sections, each is symmetric about its own midpoint (Figure 2). The midpoint of the cooling straight located at the marker 306, which coincides azimuthally with the center of the Main Injector quadrupole named Q306. Hence the section that contains the cooling straight occupies $3 / 4$ of the insert and the other section takes up $1 / 4$ of it. Because of the sectionwide mirror symmetry, there are only 4 constraints for the section with cooling straight $\left(\beta_{\mathrm{x}}=\beta_{\mathrm{y}}=24 \mathrm{~m}, \alpha_{\mathrm{x}}=\alpha_{\mathrm{y}}\right.$ $=0$ at the midpoint), 2 for the other section $\left(\alpha_{x}=\alpha_{y}=0\right.$ at the midpoint) and 2 for the insert as a whole (total phase advances). As a result, at only 8 families of quads are required. Due to the fact that the short section is only $34.58 \mathrm{~m}$ long, it is impossible to place 4 families of quads. On the other hand, one more family is needed in the long section to control maximum $\beta$ and phase advances. The process of optimization is iterative. First a solution for the long section was found using 4 out 5 families of quads. The maximum $\beta$ and phase advances of the section are adjusted through changing the drifts among the quads. The gradient of the 5th family is fixed to meet the requirement on quad strengths. Then the gradients of the 3 families and the length of a drift in the short section are varied to find a solution that satisfies the other 4 constraints. If successful, an optical solution of the insert had been found. In order to further reduce the number quad families, lengths of 2 drifts in the long section were varied and a solution was found with only 6 families of quads. Note that the last step is meaningful only for a permanent magnet beam line. The final lattice is shown in Figure 2 and Figure 3. The insert consists of 40 quads, exactly those that were available and meets all constraints mentioned above. A side product of the new lattice is that the phase advances of both planes are roughly the same, which makes the tunes of the ring $(25.425,24.415)$ as oppose to the old tunes of $(24.425$, 24.415).

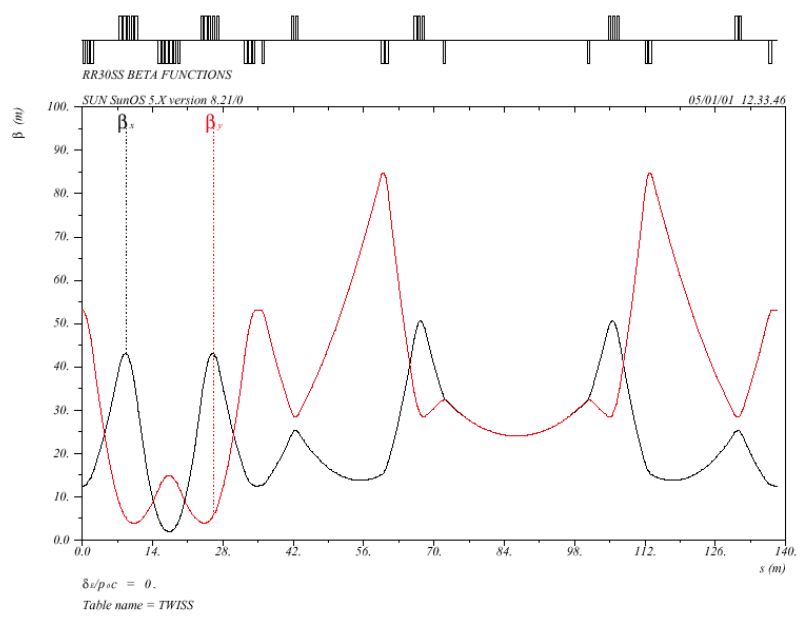

Figure 2: $\beta$-function plot of the medium- $\beta$ insert

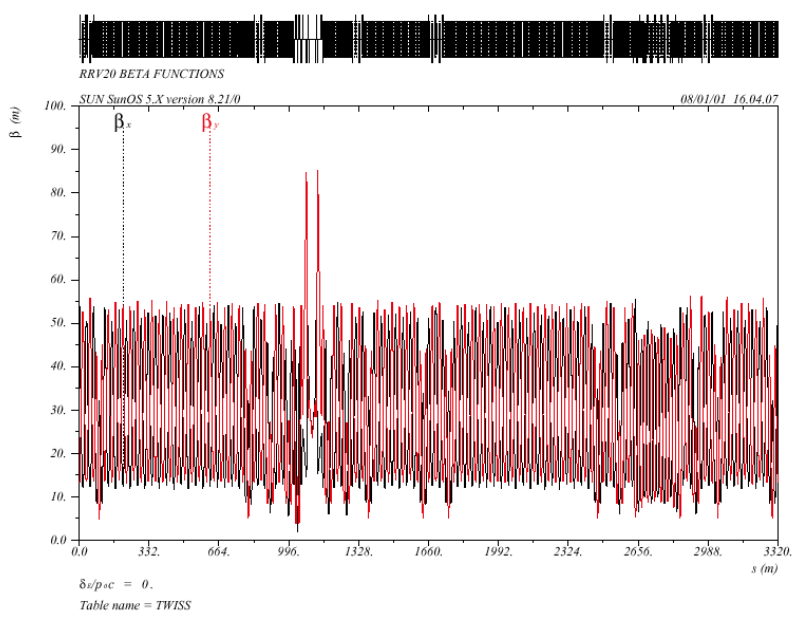

Figure 3: $\beta$-function plot of the Recycler Ring with measured quad gradient

\section{ENGINEERING DESIGN AND INSTALLATION}

One of the key issues for the cooling insert replacement was the installation time was limited to the 5 weeks during the December 2000 shutdown. The planning and staging of parts and components was a critical part in removal of the old insert and the installation of the insert on schedule.

The "as built" high-beta insert utilized ten large aperture (4.25") quads, which would not be used in the new insert, and eight lattice quads with a 3" aperture to be reused in the new insert. Of the forty quads to be installed, thirty-two could have their strength and harmonics tuned (trimmed) prior to the beginning of the shutdown, leaving only eight of the quads to be removed and tuned (trimmed) during the shutdown. This advanced quad trimming allowed a large pool of magnets to be accumulated prior to the shutdown.

The gradient strength of the quads is set to $1-2 \%$ greater than the design value by the combination of ferrite brick sizes and compensator strip quantities [7]. The integrated gradient may be precisely reduced to design value by the addition of steel washers in the corner regions on the pole centerline (See Fig. 4). Besides the integrated gradient, the normal and skew sextupole and skew octupole are controlled by the number and distribution of washers in the four corners. A complete description of the design and trimming can be found in Ref. 7.

The existing straight section had a mixture of three-inch four-inch, and elliptical beam pipe. Only the three-inch and elliptical beam pipe could be reused. All beam pipe installed in the Recycler must be hydrogen degassed prior to installation to assure the capability of vacuums in $10^{-10}$ range. The inventory of spare 3 " beam pipe that had been hydrogen degassed was minimum, so a creative combination of three inch and elliptical beam pipe had to be utilized. 
In addition to the new magnet configuration, new vacuum valves, ion pumps, BPM's, titanium sublimation pumps (TSP), and correctors were added and or moved. This involved new cabling and control crates/cards. Figure 5 shows the installation of a new quad in the straight section before its beam pipe is welded. The available resources for the removal and re-installation of all components included five riggers/welders and three vacuum technicians. The project was completed in four weeks. A fifth week was required to bake out the section of beam pipe open to air and fire the TSP's.
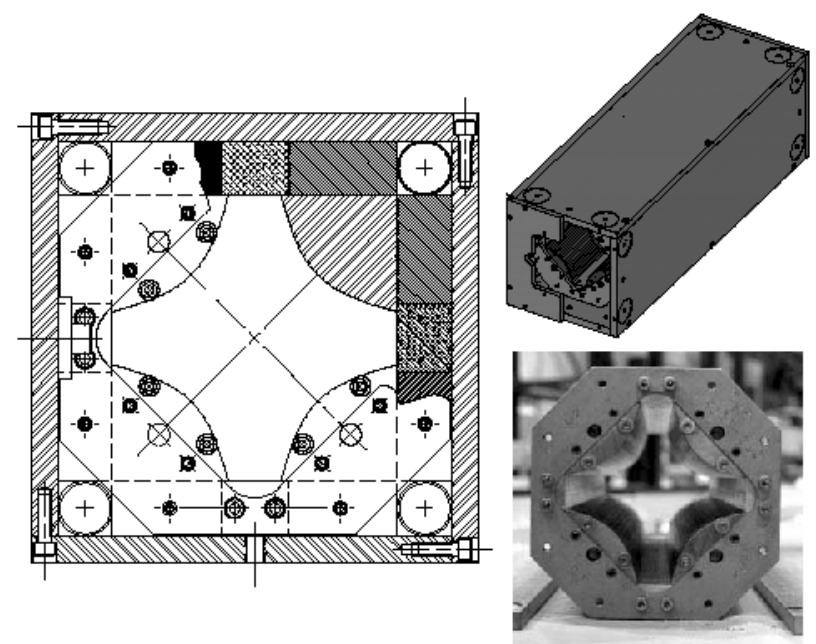

Figure 4: Drawings of the 20" long quadrupoles Note the washers in the four corners used for strength trimming

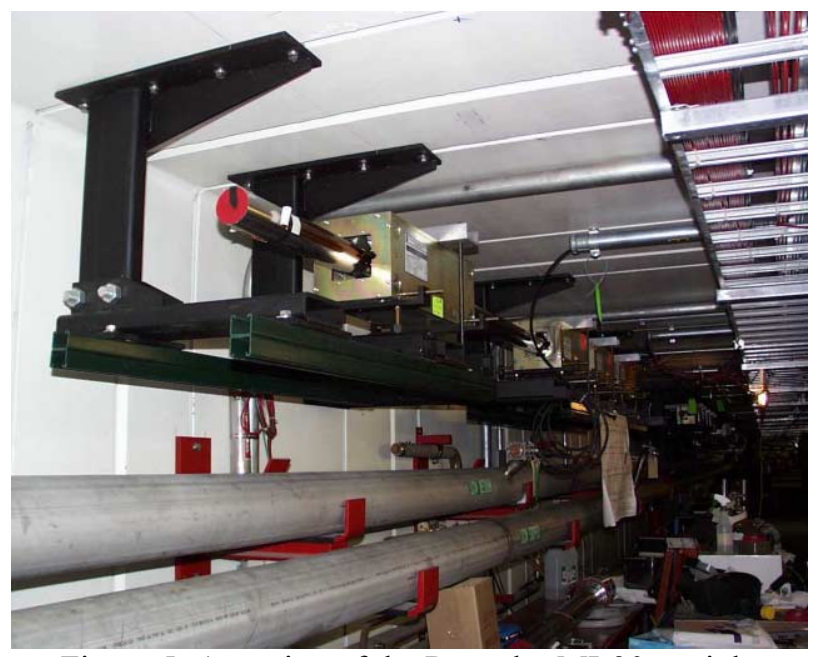

Figure 5: A section of the Recycler MI-30 straight section with the new cooling insert

\section{INITIAL PERFORMANCE}

Within three days of re-establishing beam with the new Insert the initial circulation efficiency was greater than $90 \%$. The bear machine tunes with the phase trombone quads off were measured to be $\mathrm{Q}_{\mathrm{x}}=25.456$ and $\mathrm{Q}_{\mathrm{y}}=$ 24.398 as shown in Figure 6. The expected tunes of the design lattice, incorporating all measured multipoles, is $\mathrm{Q}_{\mathrm{x}}=25.429$ and $\mathrm{Q}_{\mathrm{y}}=24.415$. This results in a shift of $\mathrm{dQ}_{\mathrm{x}} \sim+0.03$ and $\mathrm{dQ}_{\mathrm{y}} \sim-0.015$, easily within tuning range of the phase trombone.

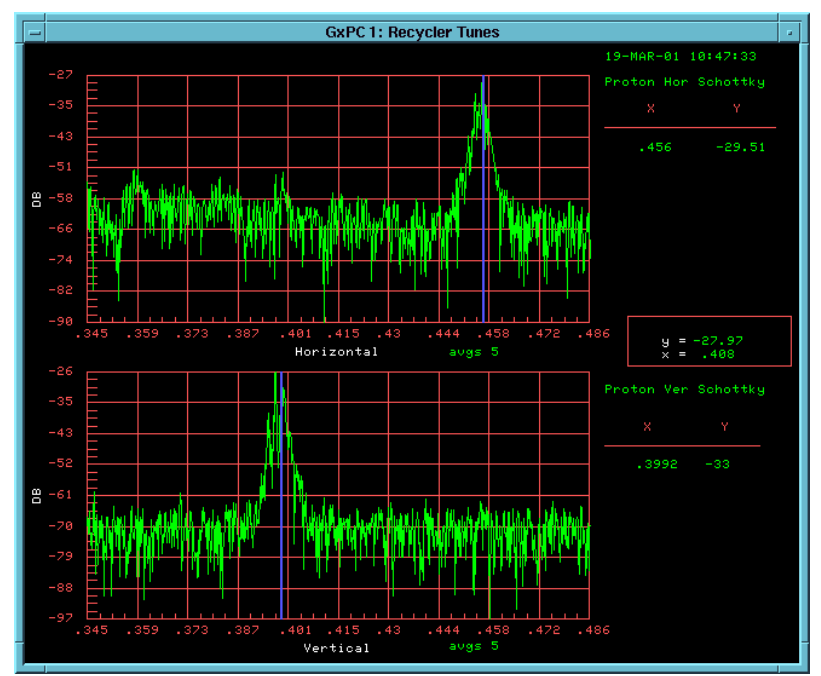

Figure 6: Tune spectra from Schottky with all tune quads in phase trombone off.

\section{CONCLUSION}

The design of the medium- $\beta$ insert has met all optical and physical constraints and it has since been successfully installed. Preliminary measurement shows that the base tunes agree with the model to better than $10^{-3}$. Tracking studies of the ring with the medium- $\beta$ insert shows the doubling of the dynamic aperture [8], which has been corroborated by improved beam lifetime [1]. Compared to the high- $\beta$ insert, the medium- $\beta$ insert reduces closed orbit distortion, maximum beam size, sensitivity to errors and, possibly, beam loss from the Main Injector stray magnetic field. The one unit tune split between the two planes should help reduce coupling. It is our believe that the medium- $\beta$ insert will prove valuable both for better electron cooling and better storage of beam.

\section{REFERENCES}

[1] $\mathrm{M}$. Hu, these proceedings.

[2] G. Jackson, FERMILAB-TM-1991 (1996).

[3] S. Mishra, these proceedings.

[4] S. Nagaitsev, these proceedings.

[5] D. E. Johnson et. al., Proc. 1997 Part. Accel. Conf., p.997 (1997).

[6] A. Burov, FERMILAB-TM-2117 (2000).

[7] B. C. Brown, et. al. , FERMILAB-Conf-97/337.

[8] M. Xiao, these proceedings. 